\section{Stepping into the virtual unknown: feasibility study of a virtual reality-based test of ocular misalignment}

N Nesaratnam ${ }^{1}, \mathrm{P}$ Thomas $^{1}$ and A Vivian

\begin{abstract}
Introduction Dissociated tests of strabismus provide valuable information for diagnosis and monitoring of ocular misalignment in patients with normal retinal correspondence. However, they are vulnerable to operator error and rely on a fixed head position. Virtual reality headsets obviate the need for head fixation, while providing other clear theoretical advantages, including complete control over the illumination and targets presented for the patient's interaction.

Purpose We compared the performance of a virtual reality-based test of ocular misalignment to that of the traditional Lees screen, to establish the feasibility of using virtual reality technology in ophthalmic settings in the future.

Methods Three patients underwent a traditional Lees screen test, and a virtual reality headset-based test of ocular motility. The virtual reality headset-based programme consisted of an initial test to measure horizontal and vertical deviation, followed by a test for torsion.
\end{abstract}

Results The pattern of deviation obtained using the virtual reality-based test showed agreement with that obtained from the Lees screen for patients with a fourth nerve palsy, comitant esotropia, and restrictive thyroid eye disease.

Conclusions This study reports the first use of a virtual reality headset in assessing ocular misalignment, and demonstrates that it is a feasible dissociative test of strabismus.

Eye (2017) 31, 1503-1506; doi:10.1038/eye.2017.97; published online 2 June 2017

\section{Introduction}

Dissociated tests of strabismus, such as the Lancaster red-green test, ${ }^{1}$ Hess screen, ${ }^{2}$ and Lees screen, ${ }^{3}$ are valuable tools in the diagnosis and monitoring of ocular misalignment in patients with normal retinal correspondence. As ophthalmologists, we often receive referrals from other specialities, notably neurology and maxillofacial surgery, requesting these tests to aid diagnosis and treatment. This case series illustrates the potential of virtual reality headsets to allow non-specialists to investigate and diagnose their patients' oculomotility disorders.

Virtual reality headsets allow complete dissociation and total control of the stimulus presented to a patient's eyes. As they are computer-driven, instructions can be given visually or audibly via the headset, and test protocols can be dynamically generated based on a patient's performance during the test. Unlike other automated tests, ${ }^{4,5}$ there is no requirement for head fixation ${ }^{4}$ as the headset moves with the patient. Therefore, virtual reality headsets offer the possibility of robust characterisation of a patient's oculomotility disorders in a non-specialist environment.

\section{Methods}

The patients presented here were part of a small pilot study to investigate the feasibility of virtual reality headsets for testing strabismus. Each patient recruited to the study underwent a traditional Lees screen test, as described by Timms, ${ }^{6}$ and a virtual reality headset-based test of ocular motility.

The test required patients to wear an Oculus Rift (commercial version, 2016) ${ }^{7}$ virtual reality headset (Figure 1). A computer displayed an image to the virtual reality headset and concurrently to a television monitor visible to the examiner. The virtual images remained fixed relative to the patient's head.

The test protocol was written in PsychoPy (version 1.83.04), ${ }^{8}$ and it consisted of an initial
Department of

Ophthalmology,

Addenbrooke's Hospital,

Cambridge, UK

Correspondence:

N Nesaratnam, Department of Ophthalmology, Addenbrooke's Hospital, Hills Road, Cambridge CB2 OQQ, UK Tel: +44 (0)7706 134672 E-mail: Nisha.n@doctors. org.uk

${ }^{1}$ These authors contributed equally to this work.

Received: 15 January 2017 Accepted in revised form: 11 April 2017 Published online: 2 June 2017 


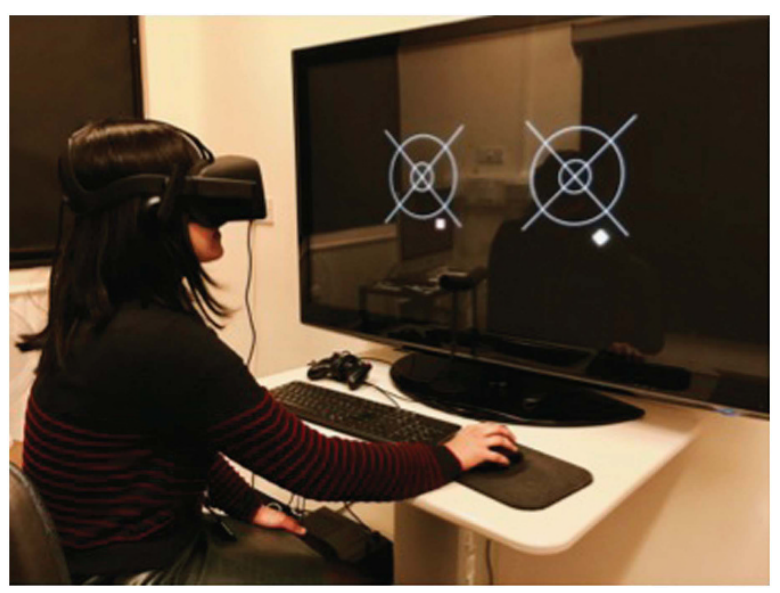

Figure 1 Photograph of head-mounted virtual reality device in use. test to measure horizontal and vertical deviation, followed by a test for torsion.

\section{Case 1}

A 47-year-old female with a known Arnold Chiari I malformation and cervical cord syrinx presented with worsening headaches, left arm weakness, and ataxia. MRI revealed enlargement of the syrinx; thus, surgical decompression of the medulla and syrinx was undertaken. Immediately following the operation, the patient developed headaches and diplopia, and orthoptic assessment revealed a fourth nerve palsy (Figure 2a), likely due to damage to the trochlear nerve intraoperatively. This finding was corroborated by the virtual reality headset-based test of ocular deviation (Figure $2 b$ ). a

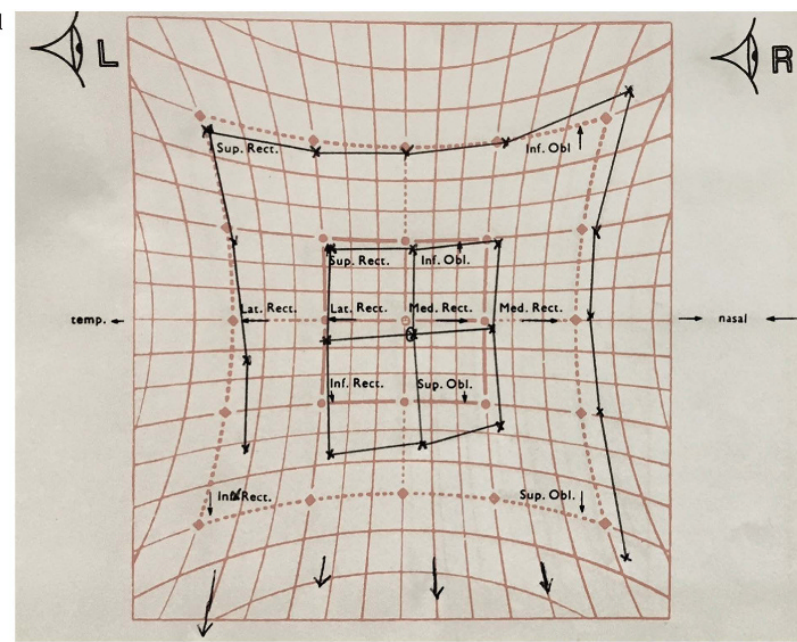

b

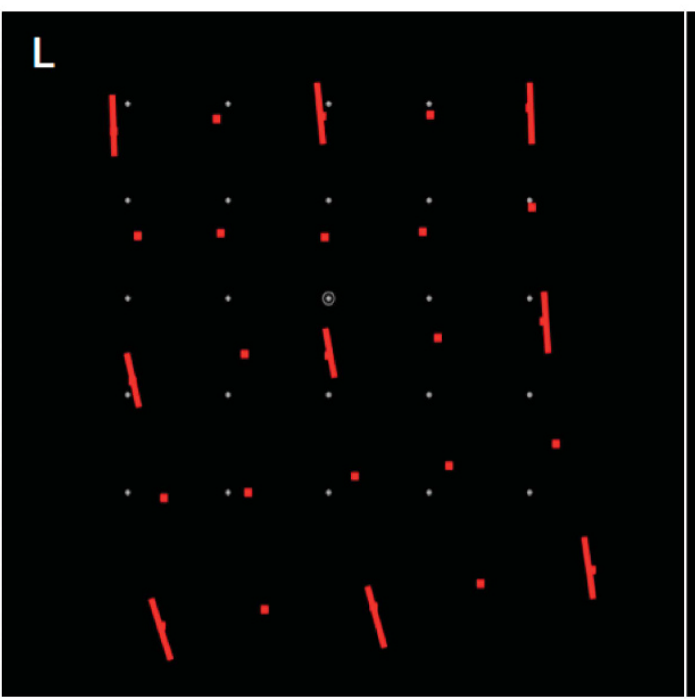

$\hat{R}$

$\mathbf{R}$
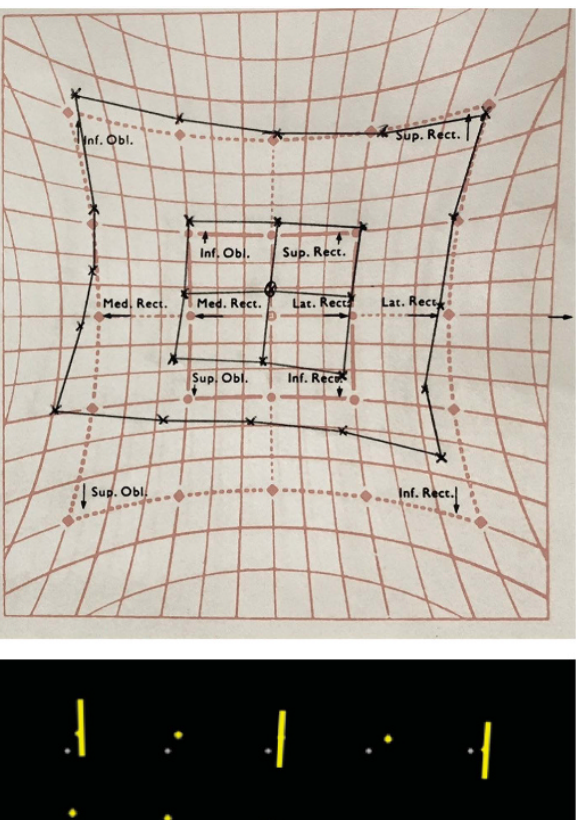

$\begin{array}{llll}- & \bullet & \bullet & :\end{array}$

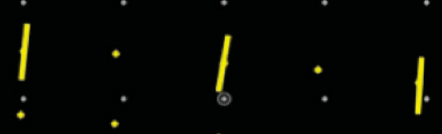

l.

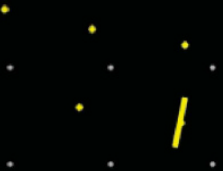

Figure 2 Pattern of ocular deviation obtained using the Lees screen (a) and virtual reality-based test (b), overlaid to illustrate good agreement between the two tests in this patient with a fourth nerve palsy. The vertical lines (b) show excyclotorsion between the two eyes (a completely vertical line means no torsion). 


\section{Case 2}

A 73-year-old male presented with intractable diplopia, secondary to thyroid eye disease. On examination, restriction of abduction was noted. MRI revealed enlargement of the inferior and medial recti bilaterally, with orbital apex crowding but no significant optic nerve a

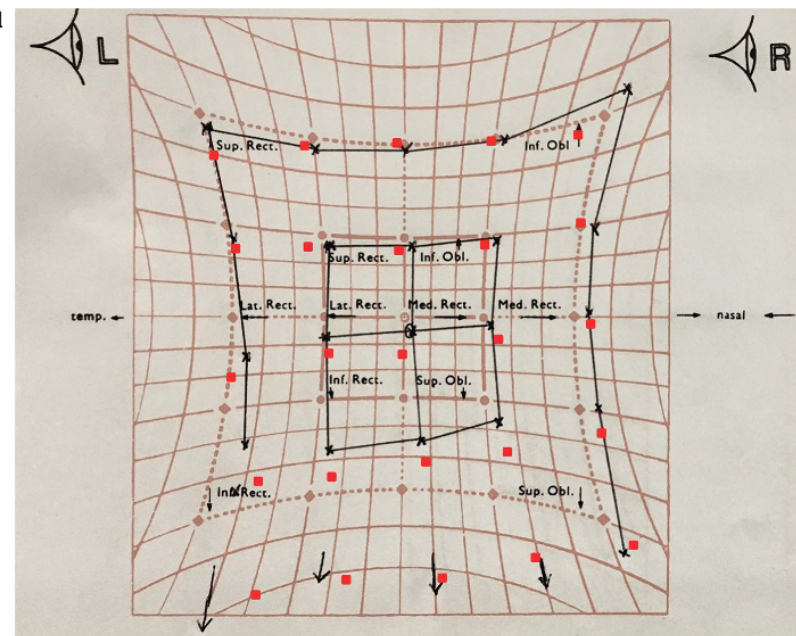

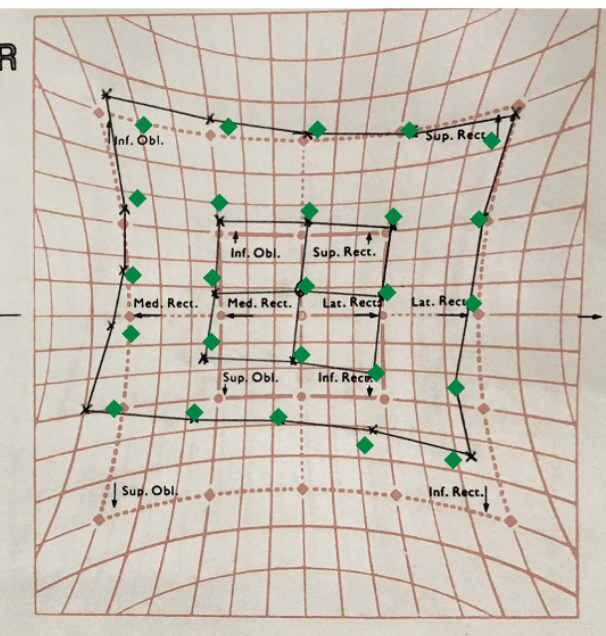

b

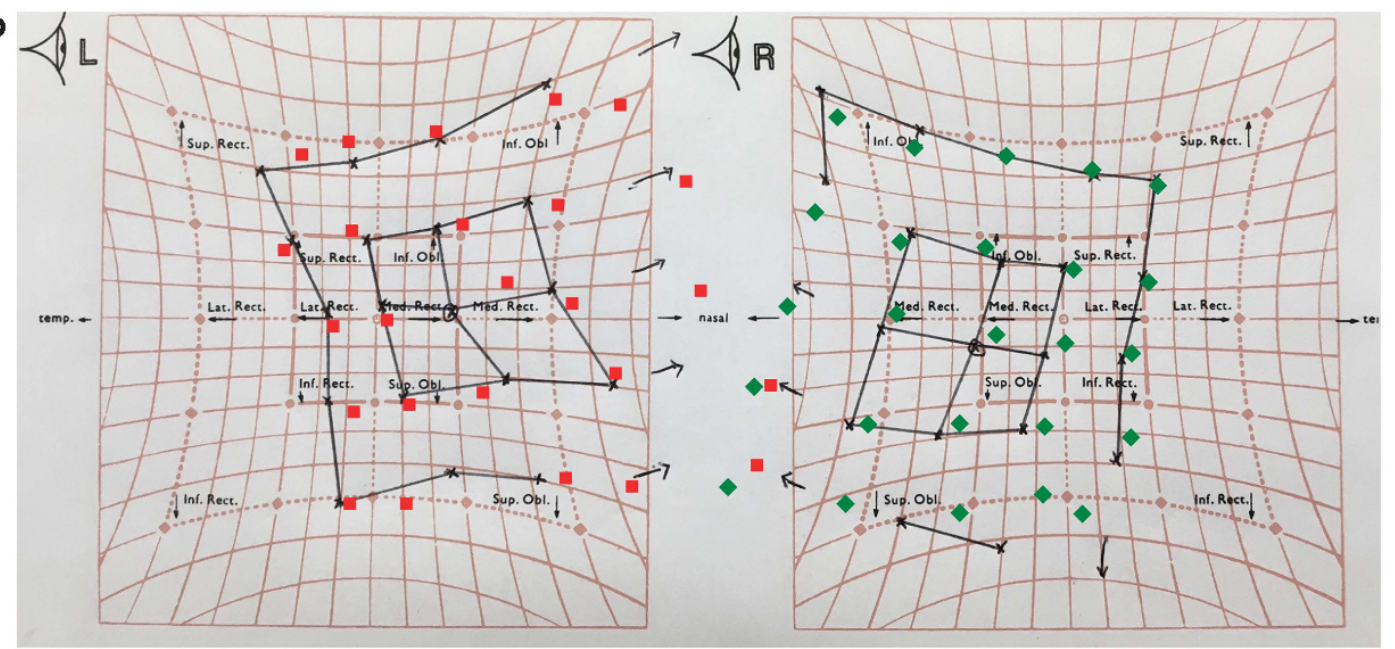

c

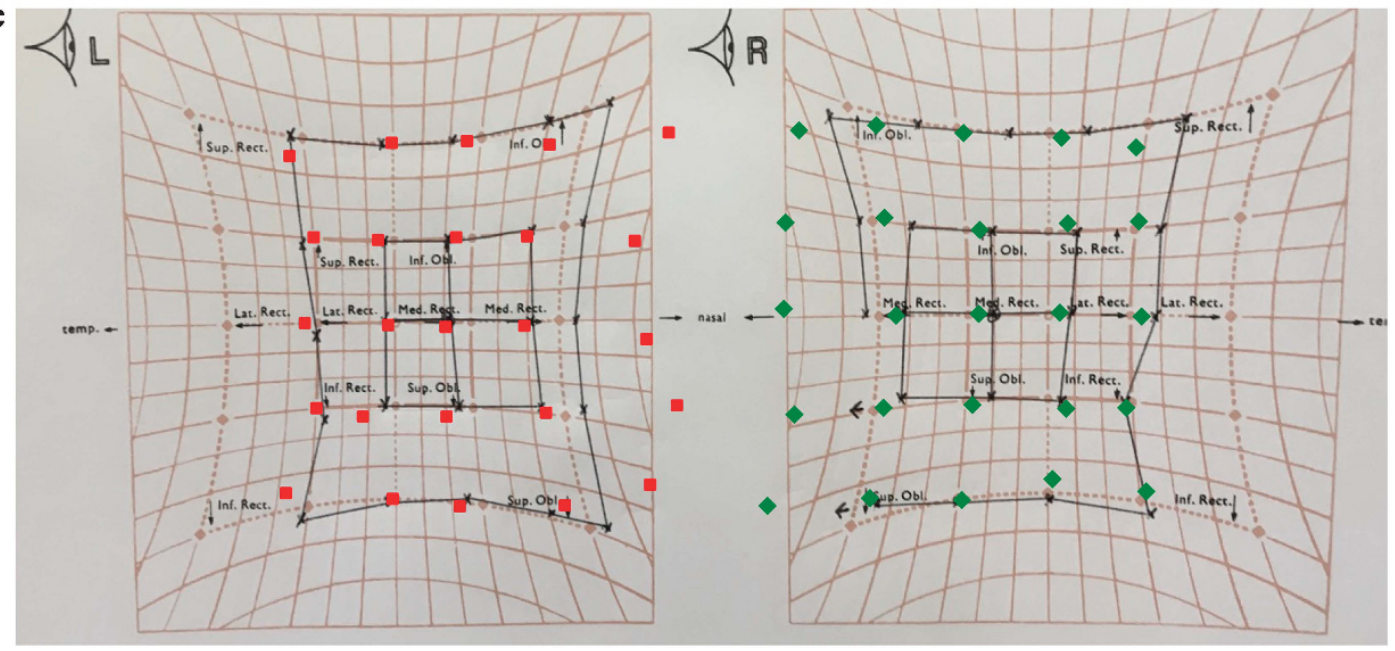

Figure 3 Overlays of patterns of ocular deviation obtained using the Lees screen (black line) and virtual reality-based test (coloured dots) for (a) fourth nerve palsy, (b) restrictive thyroid eye disease, and (c) comitant esotropia. 
stretch. TSH receptor antibodies and thyroid peroxidase antibodies were both raised.

\section{Case 3}

A 22-year-old male presented to oculomotility clinic after noticing his eyes 'drifting inwards' over the previous 2 years, and occasional diplopia when tired. There was no previous trauma or ocular history. Examination revealed a left esotropia with bilateral abduction deficit. MRI head showed no focal brain lesions and normal appearances of the orbit.

As demonstrated by the overlays in Figure 3, there is good agreement between the virtual reality-based test and Lees screen in the three cases described.

\section{Discussion}

This case series describes the first use of a virtual reality headset in assessing ocular misalignment, and demonstrates that it is a feasible dissociative test of strabismus. Comparison of results with the Lees screen test indicates good agreement across a range of common pathologies. A useful advantage of our approach is that it removes the influence of head position in yaw (face turn) and pitch (chin up/down): the position of the stimulus is coupled to the patient's head; therefore, gaze direction does not change with head movement. Head roll (tilt), however, could still affect the torsional component of our test in fourth nerve palsies, for example. Orientation tracking (gyroscope, accelerometer, and magnetometer within the headset) and position tracking (infrared microLEDs in the headset tracked by the infrared camera) are built into the headset we used, so could be used to instruct the patient to return to the neutral position if head roll creeps in, or to record the head posture in which a given measurement is made.

The low cost of virtual reality headsets opens exciting possibilities for clinical testing and automated diagnosis of patients outside of the traditional orthoptic department. In ongoing work, we are replacing our simplistic stimuli with fully realised 3D virtual realitytesting environments, in order to more accurately measure the ocular deviation encountered in the real world. We plan to add eye tracking to our current system in future clinical trials, to achieve objective assessment of ocular misalignment and field of binocular single vision.

\section{Summary}

What was known before

- Dissociated tests of strabismus provide valuable tools for diagnosis and monitoring of ocular misalignment in patients with normal retinal correspondence. However, they are vulnerable to operator error and rely on a fixed head position.

- Virtual reality headsets obviate the need for head fixation, while providing other clear theoretical advantages, including complete control over the illumination and targets presented for the patient's interaction.

What this study adds

- This case series describes the first use of a virtual reality headset in assessing ocular misalignment, and demonstrates that it is a feasible dissociative test of strabismus.

- The low cost of virtual reality headsets opens exciting possibilities for clinical testing and automated diagnosis of patients outside of the traditional orthoptic department.

\section{Conflict of interest}

The authors declare no conflict of interest.

\section{Acknowledgements}

We thank the Cambridge Eye Trust for funding the virtual reality headset and computer.

\section{References}

1 Lancaster WB. Detecting, measuring, plotting and interpreting ocular deviations. Arch Ophthalmol 1939; 22: 867-880.

2 Hess WR. I. Ein einfaches messendes Verfahren zur Motilitätsprüfung der Augen. Ophthalmologica 1916; 35: 201-219.

3 Lees VT. A new method of applying the screen test for interocular muscle balance. Br J Ophthalmol 1949; 33(1): 54-59.

4 Awadein A. A computerized version of the Lancaster red-green test. J AAPOS 2013; 17: 197-202.

5 Watts P, Nayak H, Lim MK, Ashcroft A, Al Madfai H, Palmer H. Validity and ease of use of a computerized Hess chart. J AAPOS 2011; 15: 451-454.

6 Timms C. The Lees screen test. Am Orthopt J 2006; 56: 180-183.

7 'Oculus VR' (2014). Oculus Rift. Available at http://www. oculusvr.com/rift/ (accessed 2 November 2016).

8 Peirce JW. Generating stimuli for neuroscience using PsychoPy. Front Neuroinformatics 2008; 2: 10. 